Article

\title{
Some Unified Integrals for Generalized Mittag-Leffler Functions
}

\author{
Prakash Singh ${ }^{1}\left(\mathbb{D}\right.$, Shilpi Jain ${ }^{2}$ (D) and Carlo Cattani ${ }^{3, *(\mathbb{D})}$ \\ 1 Department of Mathematics, Research Scholar, Poornima University, Jaipur 303905, India; \\ prakashsingh705@gmail.com \\ 2 Department of Mathematics, Poornima University, Jaipur 303905, India; shilpi.jain@poornima.org \\ 3 Engineering School (DEIM), University of Tuscia, 01100 Viterbo, Italy \\ * Correspondence: cattani@unitus.it
}

check for

updates

Citation: Singh, P.; Jain, S.; Cattani, C. Some Unified Integrals for Generalized Mittag-Leffler Functions. Axioms 2021, 10, 261. https:// doi.org/10.3390/axioms10040261

Academic Editor: Emil Saucan

Received: 27 September 2021 Accepted: 15 October 2021

Published: 19 October 2021

Publisher's Note: MDPI stays neutral with regard to jurisdictional claims in published maps and institutional affiliations.

Copyright: (c) 2021 by the authors. Licensee MDPI, Basel, Switzerland. This article is an open access article distributed under the terms and conditions of the Creative Commons Attribution (CC BY) license (https:/ / creativecommons.org/licenses/by/ $4.0 /)$.
Abstract: Here, we ascertain generalized integral formulas concerning the product of the generalized Mittag-Leffler function. These integral formulas are described in the form of the generalized Lauricella series. Some special cases are also presented in terms of the Wright hypergeometric function.

Keywords: wright hypergeometric functions ${ }_{p} \Psi_{q}$; generalized Lauricella series; Mittag-Leffler function; Oberhettinger's integral formula

MSC: 33B10; 33B15; 33C05; 33C20; 33C65; 33E12

\section{Introduction and Preliminaries}

There are many properties like integral formulas and differential formulas concerning a diversity of special functions; specifically, hypergeometric functions and Mittag-Leffler functions have been discussed by numerous authors [1-9]. Recently, integral formulas concerning a generalized Mittag-Leffler function have been introduced by Jain et al. [10].

Currently, we are using a product of the generalized Mittag-Leffler function to ascertain some generalized integral formulas, which was described by Prabhakar [11]:

$$
\begin{aligned}
& \prod_{i=1}^{m}\left(E_{\left(\rho_{i}\right), \zeta_{i}}^{\left(\gamma_{i}\right)}\left(z_{i}\right)\right) \equiv E_{\left(\rho_{1}\right), \zeta_{1}}^{\left(\gamma_{1}\right)}\left(z_{1}\right) E_{\left(\rho_{2}\right), \zeta_{2}}^{\left(\gamma_{2}\right)}\left(z_{2}\right) \cdots E_{\left(\rho_{m}\right), \zeta_{m}}^{\left(\gamma_{m}\right)}\left(z_{m}\right) \\
& \quad=\sum_{l_{1}=0}^{\infty} \frac{\left(\gamma_{1}\right)_{l_{1}} z_{1}^{l_{1}}}{\Gamma\left(\zeta_{1}+\rho_{1} l_{1}\right)\left(l_{1}\right) !} \sum_{l_{2}=0}^{\infty} \frac{\left(\gamma_{2}\right)_{l_{2}} z_{2}^{l_{2}}}{\Gamma\left(\zeta_{2}+\rho_{2} l_{2}\right)\left(l_{2}\right) !} \cdots \sum_{l_{m}=0}^{\infty} \frac{\left(\gamma_{m}\right)_{l_{m}} z_{m}^{l_{m}}}{\Gamma\left(\zeta_{m}+\rho_{m} l_{m}\right)\left(l_{m}\right) !},
\end{aligned}
$$

where, $\left(\zeta_{i}, \gamma_{i}, \rho_{i}, z_{i} \in \mathbb{C}, \Re\left(\rho_{i}\right)>0, i=1, \cdots, m\right)$.

Remark 1. If we set $i=1$ in (1.1), it becomes the generalized Mittag-Leffler function, due to Prabhakar [11]:

$$
E_{\rho, \zeta}^{\gamma}(z)=\sum_{l=0}^{\infty} \frac{(\gamma)_{l} z^{l}}{\Gamma(\zeta+l \rho) l !},
$$

where, $(\gamma, \rho, \zeta \in \mathbb{C}, \Re(\rho), \Re(\zeta)>0, z \in \mathbb{C})$, with $(\zeta)_{m}$ defined by the Pochhammer symbol as (see [12]):

$$
\begin{aligned}
(\zeta)_{m}: & = \begin{cases}1 & (m=0) \\
\zeta(\zeta+1) \ldots(\zeta+m-1) & (m \in \mathbb{N}:=\{1,2,3, \ldots\})\end{cases} \\
& =\frac{\Gamma(\zeta+m)}{\Gamma(\zeta)} \quad\left(\zeta \in \mathbb{C} \backslash \mathbb{Z}_{0}^{-}\right),
\end{aligned}
$$

and $\mathbb{Z}_{0}^{-}$represents the set of non-positive integers. 
The generalized Lauricella series is defined as [13]:

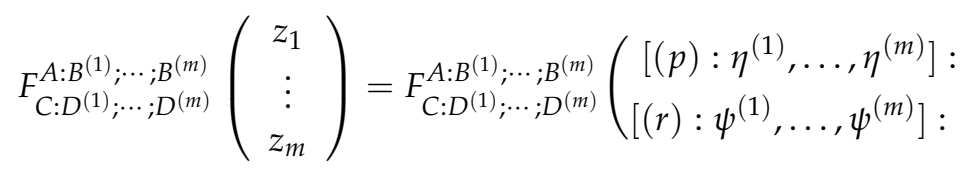

$$
\begin{aligned}
& \begin{array}{l}
{\left[(q)^{(1)}: \phi^{(1)}\right] ; \ldots ;\left[(q)^{(m)}: \phi^{(m)}\right] ;} \\
{\left[(s)^{(1)}: \delta^{(1)}\right] ; \ldots ;\left[(s)^{(m)}: \delta^{(m)}\right] ;}
\end{array} \\
& =\sum_{l_{1}, \ldots, l_{m}=0}^{\infty} \Delta\left(l_{1}, \ldots, l_{m}\right) \frac{z_{1}^{l_{1}}}{l_{1} !} \cdots \frac{z_{m}^{l_{m}}}{l_{m} !},
\end{aligned}
$$

where, for convenience,

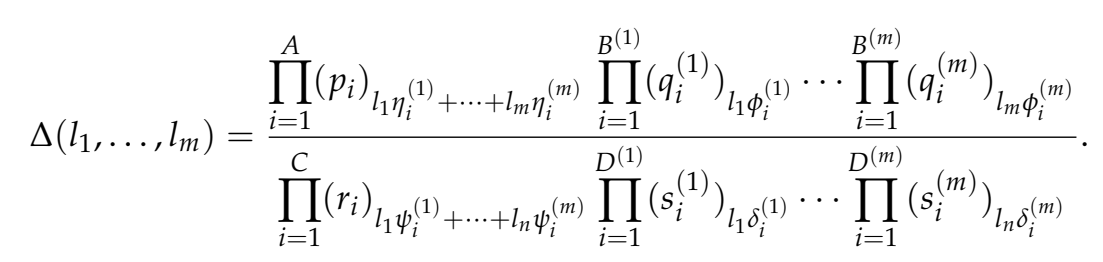

Here, we recommend $[13,14]$, for the convergence conditions of the generalized Lauricella series.

The Oberhettinger's integral formula is given by [15]:

$$
\int_{0}^{\infty} y^{\mu-1}\left(y+b+\sqrt{y^{2}+2 b y}\right)^{-\zeta} d y=2 \zeta b^{-\zeta}\left(\frac{b}{2}\right)^{\mu} \frac{\Gamma(2 \mu) \Gamma(\zeta-\mu)}{\Gamma(1+\zeta+\mu)},
$$

here $0<\Re(\mu)<\Re(\zeta)$.

The unified integral is defined by Edward as [16]:

$$
\int_{0}^{1} \int_{0}^{1}\left(v^{\prime}\right)^{v}\left(1-u^{\prime}\right)^{v-1}\left(1-v^{\prime}\right)^{\omega-1}\left(1-u^{\prime} v^{\prime}\right)^{1-v-\omega} d u^{\prime} d v^{\prime}=\frac{\Gamma(v) \Gamma(\omega)}{\Gamma(v+\omega)},
$$

here, $\Re(v)>0, \Re(\omega)>0$.

We also require the generalized hypergeometric function ${ }_{r} \psi_{s}[z]$ defined as [17]:

$$
r \psi_{s}[z]=\sum_{t=0}^{\infty} \frac{\prod_{i^{\prime}=1}^{r} \Gamma\left(a_{i^{\prime}}+\alpha_{i^{\prime}} t\right)}{\prod_{j^{\prime}=1}^{s} \Gamma\left(b_{j^{\prime}}+\beta_{j^{\prime}} t\right)} \frac{z^{t}}{t !}
$$

provided that, $r, s \in N_{0}=N \bigcup\{0\} ; a_{i^{\prime}}, b_{j^{\prime}} \in \mathbb{C} ; \alpha_{i^{\prime}}, \beta_{j^{\prime}} \in \mathbb{R} ; \alpha_{i^{\prime}}, \beta_{j^{\prime}} \neq 0 ; i^{\prime}=1, \ldots . r ; j^{\prime}=1, \ldots, s$.

The generalized Mittag-Leffler function is [18]:

$$
\begin{aligned}
& E_{\rho_{i}, \zeta}^{\gamma_{i}}\left(\frac{a_{j} y^{\prime}\left(1-x^{\prime}\right)\left(1-y^{\prime}\right)}{\left(1-x^{\prime} y^{\prime}\right)^{2}}\right)= \\
& \frac{\left(\gamma_{1}\right) l_{l_{1}} \cdots\left(\gamma_{m}\right)_{l_{m}}}{\Gamma\left(\zeta+\rho_{1} l_{1}+\cdots \rho_{m} l_{m}\right)}\left(\left(\frac{a_{1} y^{\prime}\left(1-x^{\prime}\right)\left(1-y^{\prime}\right)}{\left(1-x^{\prime} y^{\prime}\right)^{2}}\right)^{l_{1}} \frac{1}{l_{1} !} \cdots\left(\frac{a_{m} y^{\prime}\left(1-x^{\prime}\right)\left(1-y^{\prime}\right)}{\left(1-x^{\prime} y^{\prime}\right)^{2}}\right)^{l_{m}} \frac{1}{l_{m} !}\right) \\
& \text { here, } i=1, \ldots, m .
\end{aligned}
$$

\section{Main Results}

In the present paper, first, we introduce two main Theorems by using the product of the generalized Mittag-Leffler function in Equation (1). These Theorems are defined in the 
form of the series in Equation (4). We insert the product of the generalized Mittag-Leffler function into the integrand of Equation (6), to establish our main results.

Theorem 1. For the product of the generalized Mittag-Leffler function, the following integral holds:

$$
\begin{gathered}
\int_{0}^{\infty} y^{\mu-1}\left(y+b+\sqrt{y^{2}+2 b y}\right)^{-\zeta} \prod_{i=1}^{m}\left(E_{\left(\rho_{i}\right), \zeta_{i}}^{\left(\gamma_{i}\right)}\left(\frac{z_{i}}{y+b+\sqrt{y^{2}+2 b y}}\right)\right) d y \\
=2^{1-\mu} b^{\mu-\zeta} \Gamma(2 \mu) \frac{\Gamma(1+\zeta)}{\Gamma(\zeta)} \frac{\Gamma(\zeta-\mu)}{\Gamma(1+\zeta+\mu)} \frac{1}{\Gamma\left(\zeta_{1}\right) \cdots \Gamma\left(\zeta_{m}\right)} \times \\
F_{2: 1 ; \ldots ; 1}^{2: 1, \ldots ; 1}[[1+\zeta: 1, \ldots, 1],[\zeta-\mu: 1, \ldots, 1]: \\
{[\zeta: 1, \ldots, 1],[1+\zeta+\mu: 1, \ldots, 1]:} \\
\left.\left[\gamma_{1}: 1\right] ; \ldots ;\left[\gamma_{m} ; 1\right] \quad ; z_{1}, \ldots, \frac{z_{m}}{b}\right], \\
\overline{\left[\zeta_{1}, \rho_{1}\right]} ; \ldots ; \overline{\left[\zeta_{m}, \rho_{m}\right]} \quad ; \frac{b}{b},
\end{gathered}
$$

where, $\left(\mu, \zeta, z_{i} \in \mathbb{C}, y>0,(i=1, \cdots, m)\right.$ and $\left.\Re(\mu)>0, \Re(\zeta)>0, \Re(\mu)<\Re(\zeta)\right)$.

Proof of Theorem 1. Let us denote the left-hand side of Equation (10), by $\mathcal{I}$. Then use Equation (1), in the integrand of Equation (10).

We have:

$$
\begin{aligned}
\mathcal{I}= & \int_{0}^{\infty} y^{\mu-1}\left(y+b+\sqrt{y^{2}+2 b y}\right)^{-\zeta} \times \\
& \sum_{l_{1}=0}^{\infty} \frac{\left(\gamma_{1}\right)_{l_{1}}}{\Gamma\left(\zeta_{1}+\rho_{1} l_{1}\right)}\left(\frac{z_{1}}{\left(y+b+\sqrt{y^{2}+2 b y}\right)}\right)^{l_{1}} \frac{1}{l_{1} !} \cdots \times \\
& \cdots \sum_{l_{m}=0}^{\infty} \frac{\left(\gamma_{m}\right)_{l_{m}}}{\Gamma\left(\zeta_{m}+\rho_{m} l_{m}\right)}\left(\frac{z_{m}}{\left(y+b+\sqrt{y^{2}+2 b y}\right)}\right)^{l_{n}} \frac{1}{l_{m} !} d y
\end{aligned}
$$

By change of the order of summation and integration:

$$
\begin{gathered}
\mathcal{I}=\sum_{l_{1}, \cdots, l_{m}=0}^{\infty} \frac{\left(\gamma_{1}\right)_{l_{1}} \cdots\left(\gamma_{l}\right)_{l_{m}}}{\Gamma\left(\zeta_{1}+\rho_{1} l_{1}\right) \Gamma\left(\zeta_{2}+\rho_{2} l_{2}\right) \cdots \Gamma\left(\zeta_{m}+\rho_{m} l_{m}\right)} \frac{z_{1}^{l_{1}}}{l_{1} !} \cdots \frac{z_{m}^{l_{m}}}{l_{m} !} \times \\
\int_{0}^{\infty} y^{\mu-1}\left(y+b+\sqrt{y^{2}+2 b y}\right)^{-\left(\zeta+l_{1}+\cdots+l_{m}\right)} d y .
\end{gathered}
$$

By using (6), in (12), we get:

$$
\begin{aligned}
\mathcal{I}=\sum_{l_{1}, \ldots, l_{m}=0}^{\infty} \frac{\left(\gamma_{1}\right)_{l_{1}} \ldots\left(\gamma_{m}\right)_{l_{m}}}{\Gamma\left(\zeta_{1}+\rho_{1} l_{1}\right) \Gamma\left(\zeta_{2}+\rho_{2} l_{2}\right) \cdots \Gamma\left(\zeta_{m}+\rho_{m} l_{m}\right)} \times \\
\quad 2\left(\zeta+l_{1}+\ldots+l_{m}\right) b^{-\left(\zeta+l_{1}+\ldots+l_{m}\right)}\left(\frac{b}{2}\right)^{(\mu)} \frac{\Gamma(2 \mu) \Gamma\left(\zeta+l_{1}+\ldots+l_{m}-\mu\right)}{\Gamma\left(1+\zeta+\mu+l_{1}+\ldots+l_{m}\right)} \frac{\left(z_{1}\right)^{l_{1}}}{l_{1} !} \ldots \frac{\left(z_{m}\right)^{l_{m}}}{l_{m} !} .
\end{aligned}
$$


Then ordering all the non variable terms and putting $\zeta+l_{1}+\ldots+l_{m}=\frac{\Gamma\left(\zeta+l_{1}+\ldots+l_{m}+1\right)}{\Gamma\left(\zeta+l_{1}+\ldots+l_{m}\right)}$, we have:

$$
\begin{aligned}
\mathcal{I}=2^{1-\mu} b^{\mu-\zeta} \Gamma(2 \mu) \times & \left(\gamma_{1}\right)_{l_{1}} \ldots\left(\gamma_{m}\right)_{l_{m}} \\
\sum_{l_{1}, \ldots, l_{m}=0}^{\infty} \frac{\Gamma\left(\zeta+l_{1}+\cdots+l_{m}+1\right)}{\Gamma\left(\zeta_{1}+\rho_{1} l_{1}\right) \Gamma\left(\zeta_{2}+\rho_{2} l_{2}\right) \cdots \Gamma\left(\zeta_{m}+\rho_{m} l_{m}\right)} & \frac{\Gamma\left(\zeta+l_{1}+\cdots+l_{m}\right)}{\Gamma\left(\zeta+l_{1}+\cdots+l_{m}-\mu\right)} \\
& \frac{z_{1}}{\Gamma\left(1+\zeta+\mu+l_{1}+\cdots+l_{m}\right)}\left(\frac{z_{1}}{b}\right)^{l_{1}} \frac{1}{l_{1} !} \cdots\left(\frac{z_{m}}{b}\right)^{l_{m}} \frac{1}{l_{m} !} .
\end{aligned}
$$

Now, multiply and divide the above equation with $\Gamma(\zeta+1), \Gamma(\zeta), \Gamma(\zeta-\mu), \Gamma(1+\zeta+$ $\mu), \Gamma\left(\zeta_{1}\right) \cdots \Gamma\left(\zeta_{m}\right)$ and using Gamma function property as $(1+\zeta)_{l_{1}+\cdots+l_{m}}=\frac{\Gamma\left(1+\zeta+l_{1}+\cdots+l_{m}\right)}{\Gamma(1+\zeta)}$, we have:

$$
\begin{aligned}
\mathcal{I}= & 2^{1-\mu} b^{\mu-\zeta} \Gamma(2 \mu) \frac{\Gamma(1+\zeta)}{\Gamma(\zeta)} \frac{1}{\Gamma(\zeta)} \frac{\Gamma(\zeta-\mu)}{\Gamma(1+\zeta+\mu)} \frac{1}{\Gamma\left(\zeta_{1}\right) \cdots \Gamma\left(\zeta_{m}\right)} \times \\
& \sum_{l_{1}, \cdots, l_{m}=0}^{\infty} \frac{(1+\zeta)_{l_{1}+\cdots+l_{m}}(\zeta-\mu)_{l_{1}+\cdots+l_{m}}}{(\zeta)_{l_{1}+\cdots+l_{m}}(1+\zeta+\mu)_{l_{1}+\cdots+l_{m}}} \times \\
& \frac{(\gamma)_{l_{1}} \cdots(\gamma)_{l_{m}}}{\left(\zeta_{1}\right)_{\rho_{1} l_{1}} \cdots\left(\zeta_{m}\right)_{\rho_{m} l_{m}}} \frac{\left(z_{1} / b\right)^{l_{1}}}{l_{1} !} \cdots \frac{\left(z_{m} / b\right)^{l_{m}}}{l_{m} !} .
\end{aligned}
$$

By using Equation (4), in the above equation and rearranging the terms, we get our desired result, Equation (10).

Theorem 2. For the product of the generalized Mittag-Leffler function, the following integral holds:

$$
\begin{aligned}
& \int_{0}^{\infty} y^{\mu-1}\left(y+b+\sqrt{y^{2}+2 b y}\right)^{-\zeta} \prod_{i=1}^{m}\left(E_{\left(\rho_{i}\right), \zeta_{i}}^{\left(\gamma_{i}\right)}\left(\frac{y z_{i}}{y+b+\sqrt{y^{2}+2 b y}}\right)\right) d y \\
& =2^{1-\mu} b^{\mu-\zeta} \Gamma(\zeta-\mu) \frac{\Gamma(1+\zeta)}{\Gamma(\zeta)} \frac{\Gamma(2 \mu)}{\Gamma(1+\zeta+\mu)} \frac{1}{\Gamma\left(\zeta_{1}\right) \cdots \Gamma\left(\zeta_{m}\right)} \times
\end{aligned}
$$

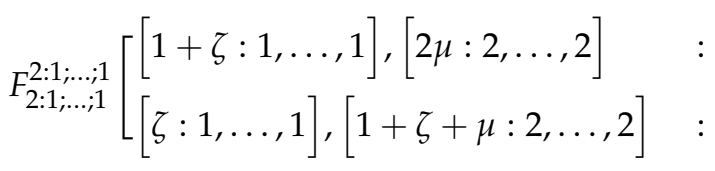

$$
\begin{aligned}
& \left.\frac{\left[\gamma_{1}: 1\right] ; \ldots ;\left[\gamma_{m} ; 1\right]}{\left[\zeta_{1}, \rho_{1}\right]} ; \ldots ; \overline{\left[\zeta_{m}, \rho_{m}\right]} \quad ; \frac{z_{1}}{2}, \ldots, \frac{z_{m}}{2}\right],
\end{aligned}
$$

where, $\left(\mu, \zeta, z_{i} \in \mathbb{C}, y>0,(i=1, \cdots, m)\right.$ and $\left.\Re(\mu)>0, \Re(\zeta)>0, \Re(\mu)<\Re(\zeta)\right)$.

Proof of Theorem 2. By following the same rule that lead to the result in Equation (10), we get our desired result, Equation (16).

Taking $i=1$ in Equations (10) and (16), following the same procedure as above, and then using Equation (8), we have the following result, which holds true for the Prabhakartype function [11]. 
Corollary 1. For the Prabhakar-type function [11], the following integral holds:

$$
\begin{array}{r}
\int_{0}^{\infty} y^{\mu-1}\left(y+b+\sqrt{y^{2}+2 b y}\right)^{-\zeta} E_{\rho, \zeta}^{\gamma}\left(\frac{z}{y+b+\sqrt{y^{2}+2 b y}}\right) d y= \\
\frac{\Gamma(2 \mu)}{\Gamma(\gamma)} 2^{(1-\mu)} b^{(\mu-\zeta)}{ }_{3} \psi_{3}\left[\begin{array}{l}
(\gamma, 1),(\zeta-\mu, 1),(\zeta+1,1) ; \\
(\zeta, \rho),(1+\zeta+\mu, 1),(\zeta, 1) ;
\end{array}\right] .
\end{array}
$$

Corollary 2. For the Prabhakar-type function [11], the following other integral also holds:

$$
\begin{array}{r}
\int_{0}^{\infty} y^{\mu-1}\left(y+b+\sqrt{y^{2}+2 b y}\right)^{-\zeta} E_{\rho, \zeta}^{\gamma}\left(\frac{y z}{y+b+\sqrt{y^{2}+2 b y}}\right) d y= \\
\frac{\Gamma(\zeta-\mu)}{\Gamma(\gamma)} 2^{(1-\mu)} b^{(\mu-\zeta)} \psi_{3}\left[\begin{array}{c}
(\gamma, 1),(\zeta+1,1),(2 \mu, 2) ; \\
(\zeta, \rho),(1+\zeta+\mu, 2),(\zeta, 1) ;
\end{array}\right] .
\end{array}
$$

Theorem 3. The unified integral associated with the generalized Mittag-Leffler-type function holds:

$$
\begin{gathered}
\int_{0}^{1} \int_{0}^{1}\left(y^{\prime}\right)^{v}\left(1-x^{\prime}\right)^{v-1}\left(1-y^{\prime}\right)^{\omega-1}\left(1-x^{\prime} y^{\prime}\right)^{1-v-\omega} E_{\rho_{i}, \zeta}^{\gamma_{i}}\left(\frac{a_{i} y\left(1-x^{\prime}\right)\left(1-y^{\prime}\right)}{\left(1-x^{\prime} y^{\prime}\right)^{2}}\right) d x^{\prime} d y^{\prime} \\
=\frac{\Gamma(v) \Gamma(\omega)}{\Gamma(v+\omega) \Gamma(\zeta)} F_{2: 0 ; \ldots ; 0}^{2: 1 ; 1}\left[\begin{array}{l}
{[v: 1, \ldots, 1],[\omega: 1, \ldots, 1]} \\
{[v+\omega: 2, \ldots, 2],\left[\zeta: \rho_{1}, \ldots, \rho_{m}\right]:}
\end{array}\right. \\
{\left[\begin{array}{c}
\left.\left.\gamma_{1}: 1\right] ; \ldots ;\left[\gamma_{m} ; 1\right] \quad ; b_{1}, \ldots, b_{m}\right] \\
-; \ldots ;-
\end{array}\right.}
\end{gathered}
$$

here, $\left(\Re(v)>0, \Re(\omega)>0, \zeta, \gamma, \rho, z_{i} \in \mathbb{C}, y>0,(i=1, \cdots, m)\right.$ and $\left.0<\Re(v)<\Re(\omega)\right)$.

Proof of Theorem 3. Let us denote the left-hand side of Equation (19) by $\mathcal{J}$. Then, we use the generalized Mittag-Leffler function in the integrand of unified integral (7). We have:

$$
\begin{aligned}
\mathcal{J}= & \int_{0}^{1} \int_{0}^{1}\left(y^{\prime}\right)^{v}\left(1-x^{\prime}\right)^{v-1} \times \\
& \left(1-y^{\prime}\right)^{\omega-1}\left(1-x^{\prime} y^{\prime}\right)^{1-v-\omega} E_{\rho_{i}, \zeta}^{\gamma_{i}}\left(\frac{a_{i} y^{\prime}\left(1-x^{\prime}\right)\left(1-y^{\prime}\right)}{\left(1-x^{\prime} y^{\prime}\right)^{2}}\right) d x^{\prime} d y^{\prime} .
\end{aligned}
$$

Then,

$$
\begin{array}{r}
\mathcal{J}=\int_{0}^{1} \int_{0}^{1}\left(y^{\prime}\right)^{v}\left(1-x^{\prime}\right)^{v-1}\left(1-y^{\prime}\right)^{\omega-1}\left(1-x^{\prime} y^{\prime}\right)^{1-v-\omega} \times \\
\sum_{l_{1}, \cdots, l_{m}=0}^{\infty} \frac{\left(\gamma_{1}\right)_{l_{1}} \cdots\left(\gamma_{m}\right)_{l_{m}}}{\Gamma\left(\zeta+\rho_{1} l_{1}+\cdots+\rho_{m} l_{m}\right)}\left(\frac{a_{1} y^{\prime}\left(1-x^{\prime}\right)\left(1-y^{\prime}\right)}{\left(1-x^{\prime} y^{\prime}\right)^{2}}\right)^{l_{1}} \frac{1}{l_{1} !} \cdots \times \\
\ldots\left(\frac{a_{m} y^{\prime}\left(1-x^{\prime}\right)\left(1-y^{\prime}\right)}{\left(1-x^{\prime} y^{\prime}\right)^{2}}\right)^{l_{m}} \frac{1}{l_{m} !} d x^{\prime} d y^{\prime} .
\end{array}
$$


Now, arranging the summation and integral part, we obtain:

$$
\begin{aligned}
\mathcal{J}=\sum_{l_{1}, \ldots, l_{m}=0}^{\infty} \frac{\left(\gamma_{1}\right) l_{l_{1}} \ldots\left(\gamma_{m}\right)_{l_{m}}}{\Gamma\left(\zeta_{1}+\rho_{1} l_{1}+\cdots+\rho_{m} l_{m}\right)} \frac{1}{l_{1} !} \cdots \frac{1}{l_{m} !} a_{i}^{l_{1}+\cdots+l_{m}} \times \\
\int_{0}^{1} \int_{0}^{1} y^{v+l_{1}+\cdots+l_{m}}\left(1-x^{\prime}\right)^{v+l_{1}+\cdots+l_{m}-1} \times \\
\left(1-y^{\prime}\right)^{\omega+l_{1}+\cdots+l_{m}-1}\left(1-x^{\prime} y^{\prime}\right)^{1-\nu-\omega+2 l_{1}+\cdots+2 l_{m}} d x^{\prime} d y^{\prime} .
\end{aligned}
$$

Now, using the unified integral (1.7), apparantly the dean has now asked about getting my thesis back...we get:

$$
\begin{array}{r}
\mathcal{J}=\sum_{l_{1}, \ldots, l_{m}=0}^{\infty} \frac{\left(\gamma_{1}\right)_{l_{1}} \cdots\left(\gamma_{m}\right)_{l_{m}}}{\Gamma\left(\zeta_{1}+\rho_{1} l_{1}+\cdots+\rho_{m} l_{m}\right)} \frac{1}{l_{1} !} \cdots \frac{1}{l_{m} !} a_{i}^{l_{1}+\cdots+l_{m}} \times \\
\frac{\Gamma\left(v+l_{1}+\cdots+l_{m}\right) \Gamma\left(\omega+l_{1}+\cdots+l_{m}\right)}{\Gamma\left(v+2 l_{1}+\cdots+2 l_{m}\right)} .
\end{array}
$$

Multiply and divide the above equation with $\Gamma(v), \Gamma(\omega), \Gamma(v+\omega), \Gamma(\zeta)$ and using the Gamma function property as $(v)_{l_{1}+\cdots+l_{m}}=\frac{\Gamma\left(v+l_{1}+\cdots+l_{m}\right)}{\Gamma(v)}$.

We get:

$$
\begin{array}{r}
\mathcal{J}=\frac{\Gamma(v) \Gamma(\omega)}{\Gamma(v+\omega) \Gamma(\zeta)} \sum_{l_{1}, \ldots, l_{m}=0}^{\infty} \frac{\left(\gamma_{1}\right)_{l_{1}} \ldots\left(\gamma_{m}\right)_{l_{m}}}{\Gamma\left(\zeta_{1}+\rho_{1} l_{1}+\cdots+\rho_{m} l_{m}\right)} \times \\
\frac{(v)_{l_{1}+\cdots+l_{m}}(\omega)_{l_{1}+\cdots+l_{m}}}{(v+\omega)_{2 l_{1}+\cdots+2 l_{m}}} \frac{\left(a_{1}\right)^{l_{1}}}{l_{1} !} \cdots \frac{\left(a_{m}\right)^{l_{m}}}{l_{m} !} .
\end{array}
$$

Then, by using the definition of the Lauricella series (4), in the above equation and rearranging the terms, we get our desired result, Theorem 3.

Theorem 4. The unified integral associated with the multiple generalized Mittag-Leffler-type function holds:

$$
\begin{aligned}
& \int_{0}^{1} \int_{0}^{1}\left(y^{\prime}\right)^{v}\left(1-x^{\prime}\right)^{\nu-1}\left(1-y^{\prime}\right)^{\omega-1}\left(1-x^{\prime} y^{\prime}\right)^{1-v-\omega} \prod_{i=1}^{m} E_{\rho_{i}, \zeta_{i}}^{\gamma_{i}}\left(\frac{a_{i} y\left(1-x^{\prime}\right)\left(1-y^{\prime}\right)}{\left(1-x^{\prime} y^{\prime}\right)^{2}}\right) d x^{\prime} d y^{\prime} \\
& =\frac{\Gamma(v) \Gamma(\omega)}{\Gamma(v+\omega) \Gamma\left(\zeta_{1}\right) \cdots \Gamma\left(\zeta_{m}\right)} F_{1: \rho_{1} ; \ldots ; \rho_{m}}^{2: 1, \ldots ; 1}[[v+\omega: 2, \ldots, 2] \quad: \\
& \left.\frac{\left[\gamma_{1}: 1\right] ; \ldots ;\left[\gamma_{m} ; 1\right]}{\left[\zeta_{1}, \rho_{1}\right]} ; \ldots ; \overline{\left[\zeta_{m}, \rho_{1}\right]} \quad ; a_{1}, \ldots, a_{m}\right],
\end{aligned}
$$

here, $\left(\operatorname{Re}(v)>0, \operatorname{Re}(\omega)>0, \zeta, \gamma, \rho, z_{i} \in \mathbb{C}, y>0,(i=1, \cdots, m)\right.$ and $\left.0<\Re(v)<\Re(\omega)\right)$.

Proof of Theorem 4. Let us denote the left-hand side of Equation (25) by $\mathcal{K}$. Then, we use the generalized Mittag-Leffler function in the integrand of unified integral in Equation (7). We have:

$$
\begin{array}{r}
\mathcal{K}=\int_{0}^{1} \int_{0}^{1}\left(y^{\prime}\right)^{v}\left(1-x^{\prime}\right)^{v-1}\left(1-y^{\prime}\right)^{\omega-1}\left(1-x^{\prime} y^{\prime}\right)^{1-v-\omega} \times \\
\prod_{i=1}^{m} E_{\rho_{i}, \zeta_{i}}^{\gamma_{i}}\left(\frac{a_{i} y^{\prime}\left(1-x^{\prime}\right)\left(1-y^{\prime}\right)}{\left(1-x^{\prime} y^{\prime}\right)^{2}}\right) d x^{\prime} d y^{\prime}
\end{array}
$$


By the change of the order of summation and integration, we have:

$$
\begin{aligned}
\mathcal{K}= & \int_{0}^{1} \int_{0}^{1}\left(y^{\prime}\right)^{v}\left(1-x^{\prime}\right)^{v-1}\left(1-y^{\prime}\right)^{\omega-1}\left(1-x^{\prime} y^{\prime}\right)^{1-v-\omega} \times \\
& \sum_{l_{1}, \cdots, l_{m}=0}^{\infty} \frac{\left(\gamma_{1}\right)_{1} \cdots\left(\gamma_{m}\right)_{l_{m}}}{\Gamma\left(\zeta_{1}+l_{1} \rho_{1}\right) \cdots \Gamma\left(\zeta_{m}+l_{m} \rho_{m}\right)} \times \\
& \left(\left(\frac{a_{1} y^{\prime}\left(1-x^{\prime}\right)\left(1-y^{\prime}\right)}{\left(1-x^{\prime} y^{\prime}\right)^{2}}\right)^{l_{1}} \frac{1}{l_{1} !} \cdots\left(\frac{a_{m} y^{\prime}\left(1-x^{\prime}\right)\left(1-y^{\prime}\right)}{\left(1-x^{\prime} y^{\prime}\right)^{2}}\right)^{l_{m}} \frac{1}{l_{m} !}\right) d x^{\prime} d y^{\prime} .
\end{aligned}
$$

We obtain the following expression:

$$
\begin{array}{r}
\mathcal{K}=\sum_{l_{1}, \ldots, l_{m}=0}^{\infty} \frac{\left(\gamma_{1}\right)_{l_{1}} \ldots\left(\gamma_{m}\right)_{l_{m}}}{\Gamma\left(\zeta_{1}+l_{1} \rho_{1}\right) \cdots \Gamma\left(\zeta_{m}+l_{m} \rho_{m}\right)} \frac{1}{l_{1} !} \cdots \frac{1}{l_{m} !} a_{i}^{l_{1}+\cdots+l_{m}} \times \\
\int_{0}^{1} \int_{0}^{1}\left(y^{\prime}\right)^{v+l_{1}+\cdots+l_{m}}\left(1-x^{\prime}\right)^{v+l_{1}+\cdots+l_{m}-1} \times \\
\left(1-y^{\prime}\right)^{\omega+l_{1}+\cdots+l_{m}-1}\left(1-x^{\prime} y^{\prime}\right)^{1-v-\omega+2 l_{1}+\cdots+2 l_{m}} d x^{\prime} d y^{\prime} .
\end{array}
$$

Now, using the unified integral of Equation (7). We obtained the following:

$$
\begin{array}{r}
\mathcal{K}=\sum_{l_{1}, \ldots, l_{m}=0}^{\infty} \frac{\left(\gamma_{1}\right)_{l_{1}} \cdots\left(\gamma_{m}\right)_{l_{m}}}{\Gamma\left(\zeta_{1}+l_{1} \rho_{1}\right) \cdots \Gamma\left(\zeta_{m}+l_{m} \rho_{m}\right)} \frac{1}{l_{1} !} \cdots \frac{1}{l_{m} !} a_{i}^{l_{1}+\cdots+l_{m}} \times \\
\frac{\Gamma\left(v+l_{1}+\cdots+l_{m}\right) \Gamma\left(\omega+l_{1}+\cdots+l_{m}\right)}{\Gamma\left(v+2 l_{1}+\cdots+2 l_{m}\right)} .
\end{array}
$$

Multiply and divide the above equation with $\Gamma(v), \Gamma(\omega), \Gamma(v+\omega), \Gamma\left(\zeta_{1}\right) \cdots \Gamma\left(\zeta_{m}\right)$, and using the Gamma function property as $(v)_{l_{1}+\cdots+l_{m}}=\frac{\Gamma\left(v+l_{1}+\cdots+l_{m}\right)}{\Gamma(v)}$.

We have:

$$
\begin{aligned}
\mathcal{K}=\frac{\Gamma(v) \Gamma(\omega)}{\Gamma(v+\omega) \Gamma\left(\zeta_{1}\right) \cdots \Gamma\left(\zeta_{m}\right)} \\
\qquad \sum_{l_{1}, \ldots, l_{m}=0}^{\infty} \frac{\left(\gamma_{1}\right)_{l_{1}} \cdots\left(\gamma_{m}\right)_{l_{m}}}{\left(\zeta_{1}\right)_{l_{1} \rho_{1}} \cdots\left(\zeta_{m}\right)_{l_{m} \rho_{m}}} \frac{(v)_{l_{1}+\cdots+l_{m}}(\omega)_{l_{1}+\cdots+l_{m}}}{(v+\omega)_{2 l_{1}+\cdots+2 l_{m}}} \frac{\left(a_{1}\right)^{l_{1}}}{l_{1} !} \cdots \frac{\left(a_{m}\right)^{l_{m}}}{l_{m} !} .
\end{aligned}
$$

Then, we use the definition of the Lauricella series (Equation (4)) in the above equation, and, rearranging the terms, we get our desired result, Theorem 4 .

Remark 2. Taking $i=1$ in Equation (19), following the same procedure as above, and using Equation (8), we have the following result, which holds true for the Prabhakar-type function [11], as follows:

$$
\begin{aligned}
\int_{0}^{1} & \int_{0}^{1}\left(y^{\prime}\right)^{v}\left(1-x^{\prime}\right)^{v-1}\left(1-y^{\prime}\right)^{\omega-1}\left(1-x^{\prime} y^{\prime}\right)^{(1-v-\omega)} E_{\rho, \zeta}^{\gamma}\left(\frac{a y^{\prime}(1-x)\left(1-y^{\prime}\right)}{\left(1-x^{\prime} y^{\prime}\right)^{2}}\right) d x^{\prime} d y^{\prime} \\
& =\frac{1}{\Gamma(\gamma)^{3}} \psi_{2}\left[\begin{array}{c}
(\gamma, 1),(v, 1),(\omega, 1) ; \\
(v+\omega, 2),(\zeta, \rho) ;
\end{array}\right] .
\end{aligned}
$$

\section{Concluding Remark}

We conclude our analysis by remarking that the results presented in this article are new and important for the class of Mittag-Leffler functions. By choosing different values of parameters, we can extract several sub-results from our main results. Further research will focus on basic applications and examples of these results for various research areas. 
Author Contributions: Conceptualization, P.S. and S.J.; methodology, C.C.; software, S.J. and P.S.; validation, S.J., P.S. and C.C.; investigation, C.C.; formal analysis, C.C. and S.J.; resources, P.S.; data writing-original draft preparation, S.J.; writing—review and editing, S.J., P.S. and C.C.; visualization, P.S.; funding acquisition, P.S., C.C. All authors have read and agreed to the published version of the manuscript.

Funding: Shilpi Jain is very thankful to the funding agency SERB (project number: MTR/2017/000194) for providing necessary financial support for the present study.

Institutional Review Board Statement: Not applicable.

Informed Consent Statement: Not applicable.

Data Availability Statement: Not applicable.

Acknowledgments: The authors would like to thank the anonymous referees for their careful reading of this manuscript and also for their constructive suggestions, which considerably improved the article. Shilpi Jain is very thankful to SERB (project number: MTR/2017/000194) for providing the necessary facility.

Conflicts of Interest: The authors declare no conflict of interest.

\section{References}

1. Agarwal, P.; Choi, J. Certain unified integrals associated with Bessel functions. Bound. Value Probl. 2013, 1, 1-9.

2. Srivastava, H.M.; Agarwal, P.; Jain, S. Generating functions for the generalized Gauss hypergeometric functions. Commun. Appl. Math. Comput. 2014, 247, 348-352. [CrossRef]

3. Agarwal, P.; Al-Mdallal, Q.; Cho, Y.J.; Jain, S. Fractional differential equations for the generalized Mittag-Leffler function. Adv. Differ. Equ. 2018, 1, 1-8. [CrossRef]

4. Agarwal, R.P.; Agarwal, P.; Ruzhansky, M. (Eds.) Special Functions and Analysis of Differential Equations, 1st ed.; Chapman and Hall/CRC Press: Boca Raton, FL, USA, 2020.

5. Choi, J.; Agarwal, P. Certain unified integrals involving a product of Bessel functions of the first kind. Honam Math. J. 2013, 35, 667-677. [CrossRef]

6. Choi, J.; Agarwal, P. A note on fractional integral operator associated with multiindex Mittag-Leffler functions. Filomat 2016, 30, 1931-1939. [CrossRef]

7. Agarwal, P.; Chand, M.; Baleanu, D.; Jain, S. On the solutions of certain fractional kinetic equations involving k-Mittag-Leffler function. Adv. Differ. Equ. 2018, 1, 1-13. [CrossRef]

8. Agarwal, P.; Nieto, J.J. Some fractional integral formulas for the Mittag-Leffler type function with four parameters. Open Math. 2015, 13, 537-546. [CrossRef]

9. Goyal, R.; Agarwal, P.; Momami, S.; Rassias, M.T. An Extension of Beta Function by Using wiman's function. Axioms 2021, 10, 187. [CrossRef]

10. Jain, S.; Agarwal, R.P.; Agarwal, P.; Singh, P. Certain Unified Integrals Involving a Multivariate Mittag-Leffler Function. Axioms 2021, 10, 81. [CrossRef]

11. Prabhakar, T.R. A singular integral equation with a generalized Mittag-Leffler function in the kernel. Yokohama Math. J. 1971, 19, 7-15.

12. Srivastava, H.M.; Choi, J. Zeta and q-Zeta Functions and Associated Series and Integrals; Elsevier Science Publishers: Amsterdam, The Netherlands; London, UK; New York, NY, USA, 2012.

13. Srivastava, H.M.; Daoust, M.C. A note on the convergence of Kampé de Fériet's double hypergeometric series. Math. Nachr. 1985, 53, 151-159. [CrossRef]

14. Srivastava, H.M.; Exton, H. A generalization of the Weber-Schafheitlin integral. J. Reine Angew. 1979, 309, 1-6.

15. Oberhettinger, F. Tables of Mellin Transforms; Springer: New York, NY, USA, 1974.

16. Khan, W.A.; Khan, I.; Singh, B.M. On unified integral associated with the generalized Mittag-Leffler function. Glob. J. Pure Appl. Math. 2017, 13, 4415-4424.

17. Rainville, E.D. Special Functions; Macmillan: New York, NY, USA, 1960.

18. Saxena, R.K.; Kalla, S.L. Solutions of Volterra-type integro-differential equations with a generalized Lauricella confluent hypergeometric function in the kernels. J. Math. Math. Sci. 2005, 8, 1155-1170. [CrossRef] 